\title{
A rare and life-threatening cause of epistaxis
}

\author{
Rory McDonald, Anant Piyush Patel, Claudine Horrocks
}

Department of ENT, Head \& Neck Surgery, Peterborough City Hospital, Peterborough, UK

\section{Correspondence to} Anant Piyush Patel, anantpatel86@gmail.com

Accepted 6 December 2015

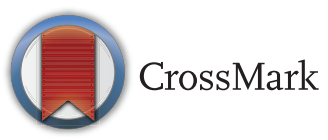

To cite: McDonald $R$, Patel AP, Horrocks C. BMJ Case Rep Published online: [please include Day Month Year] doi:10.1136/bcr-2015213528

\section{SUMMARY}

Epistaxis is a potentially life-threatening condition that can compromise a patient's airway, breathing and circulation. Early recognition of underlying bleeding diatheses that are amenable to medical therapy is imperative in order for timely treatment and to reduce morbidity. We present a case in which acquired haemophilia was diagnosed in an 83-year-old man presenting with seemingly uncomplicated epistaxis. The patient suffered multisite haemorrhages and haemodynamic compromise before definitive management with monoclonal antibody therapy. The case highlights the difficulties in managing this rare condition. It also emphasises the importance of clotting assay analysis in patients who present with epistaxis in the absence of an underlying anticoagulant.

\section{BACKGROUND}

Epistaxis is common and will affect over half the population at least once in their lifetime. ${ }^{1}$ Epistaxis can be divided on the basis of anatomical origin, with the majority being classified as 'anterior', arising from an anastomotic network of vessels in the anteroinferior part of the nasal septum known as Little's Area. The majority of these bleeds are self-limiting or will settle with simple first aid measures. However, a minority will require admission to hospital for nasal packing, and possibly operative intervention. Posterior bleeds are typically more profuse and present a greater risk of airway compromise and aspiration, yet the majority of these can also be managed non-operatively. Underlying causes can be local (including trauma, inflammation or topical medication use) or systemic (congenital or acquired blood dyscrasias and use of oral anticoagulants). A significant proportion of patients admitted to hospital with epistaxis will be taking oral anticoagulants such as warfarin, or novel alternatives including dabigatran and rivaroxaban. It is far less common to encounter adults with acquired coagulopathies. Acquired haemophilia is one such condition that is rare, has a high mortality rate, and presents diagnostic and management challenges.

\section{CASE PRESENTATION}

An 83-year-old man with no known bleeding diathesis and not on anticoagulant or antiplatelet therapy, presented to the emergency department, with unilateral epistaxis. He reported intermittent epistaxis since his elective inguinal hernia repair 1 month previously-a procedure that had been complicated by a large scrotal and groin haematoma requiring a return to theatre and multiple blood transfusions. Other medical history included Parkinson's disease with hypotension. At the time of admission, the patient was taking oral co-amoxiclav for a urinary tract infection (UTI).

A clear bleeding point could not be identified and haemorrhage control was achieved with anterior inflatable nasal packing $(5.5 \mathrm{~cm}$ Rapid Rhino, Smith and Nephew, London, UK), and the patient was admitted to the Otolaryngology ward. He was haemodynamically stable throughout. Coagulation assays revealed normal prothrombin time, and normal fibrinogen and platelet count, but derangement of activated partial thromboplastin time (APTT) ratio at 3.20. Analysis of historical laboratory results from his previous elective admission showed a slightly raised APTT ratio of 1.38, 1 month previously. At that time, despite requiring an urgent return to theatre and transfusion, the derangement was attributed to the use of prophylactic low molecular weight heparin (LMWH) and was not further investigated.

On advice from a haematology specialist, assays for lupus anticoagulant and intrinsic pathway factors were sent on the day of admission. After $24 \mathrm{~h}$, the patient had not re-bled and had a stable haemoglobin level. He was discharged home after removal of the pack, with an early haematology out-patient follow-up.

Unfortunately, he returned 1 day later with recurrent severe epistaxis, again requiring nasal packing. On this occasion, haemostasis was achieved in the emergency department, with bilateral $7.5 \mathrm{~cm}$ inflatable packs. The patient's detailed haematological assays now became available, revealing a lack of correction of APTT after mixing patient plasma with pooled normal plasma, a factor VIII level of $<1 \mathrm{IU} / \mathrm{dL}$ (normal range 50-150) and Factor VIII inhibitor levels of 64 Bethesda Units. These results revealed the diagnosis of acquired 'autoimmune' haemophilia with a high inhibitor level. Nasal packs were left in situ, and the patient was started on oral prednisolone (40 mg daily) and tranexamic acid with omeprazole for gastric protection. Oral antibiotics were continued for treatment of UTI. These antibiotics also served as prophylaxis for the indwelling nasal packs, which is our practice when these are used over $24 \mathrm{~h}$.

On day three of admission, the patient reported of groin pain and fatigue, and became haemodynamically unstable. He had a tender right flank and fullness in the right iliac fossa. Blood results revealed an acute haemoglobin drop of $32 \mathrm{~g} / \mathrm{L}$ $(102 \mathrm{~g} / \mathrm{L}$ to $70 \mathrm{~g} / \mathrm{L})$ and acute kidney injury (creatinine rise from $103 \mu \mathrm{mol} / \mathrm{L}$ to $219 \mu \mathrm{mol} / \mathrm{L}$ ). The patient was aggressively resuscitated with crystalloid, and transfused with packed red blood cells and recombinant human factor VIIa (rFVIIa). Antibiotics were changed to the intravenous route 
to cover possible chest sepsis, and prednisolone was increased to $1 \mathrm{mg} / \mathrm{kg}$ (80 mg). CT of the chest, abdomen and pelvis was performed, as the source of suspected haemorrhage could not be identified clinically. Contrast was not used in view of the significant acute kidney injury. The scan demonstrated a large haematoma in the right retroperitoneal region, involving the iliacus muscle, with maximal dimensions of $9 \times 9 \times 20 \mathrm{~cm}$ (figure 1). After surgical review, it was decided to treat this haemorrhage conservatively due to anaesthetic risk and the underlying aetiology. The patient received a total of 14 units of blood and four further units of factor VIIa over the coming days. Intravenous co-amoxiclav was continued for 4 days as prophylaxis for the retroperitoneal collection before being stepped down to oral antibiotics to continue to cover for the nasal packing.

The nasal packs were removed on day 5 , but the patient had further epistaxis overnight, and a unilateral inflatable nasal pack coated in antibacterial ointment (Bactroban, GlaxoSmithKline, London, UK) was inserted. This was left for a further 3 days during which time the patient had no significant epistaxis. On day 9, this nasal pack was successfully removed after having been deflated for $24 \mathrm{~h}$, and there was no evidence of active epistaxis or intra-abdominal bleeding over the coming week.

Two weeks into his admission, due to failure of factor VIII levels to recover significantly with high dose oral steroids, treatment with rituximab was started. Rituximab is a monoclonal antibody that targets B-cells and is used in a variety of autoimmune disorders. Its initial infusion gave promising results with climbing factor VIII levels (figure 2). Unfortunately, several days later the patient had one further episode of unilateral epistaxis on the same side as before, and was packed by the duty medical team, but at 3 weeks, after his second rituximab infusion, the nasal packing was successfully removed and after a period of observation the patient was discharged home. The final rituximab infusions were given at weekly intervals on an outpatient basis.

\section{OUTCOME AND FOLLOW-UP}

At 3 months postdischarge, the patient has suffered no further episodes of epistaxis or other bleeding. Clotting studies and factor VIII levels are back to within normal range and his haemoglobin remains stable. Oral steroids have been weaned and discontinued and the patient has been discharged from the haematology clinic.

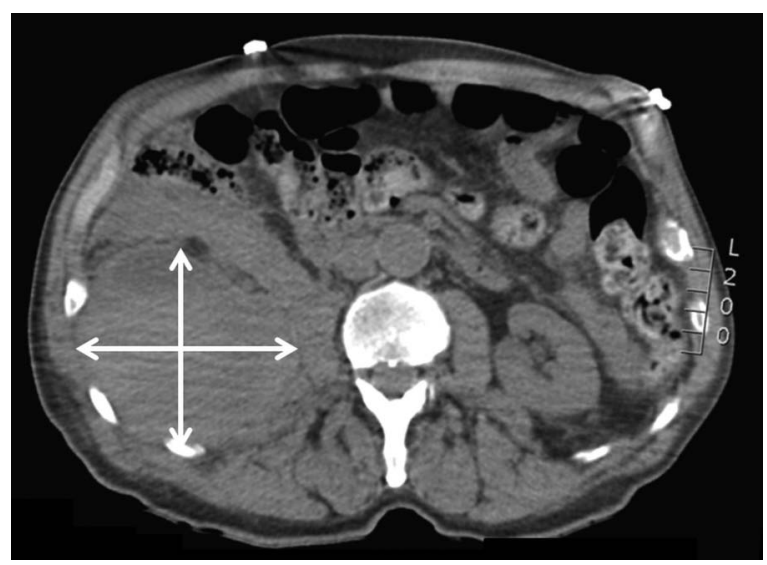

Figure 1 Axial CT image demonstrating large haematoma (white arrows) in the right retroperitoneal region.

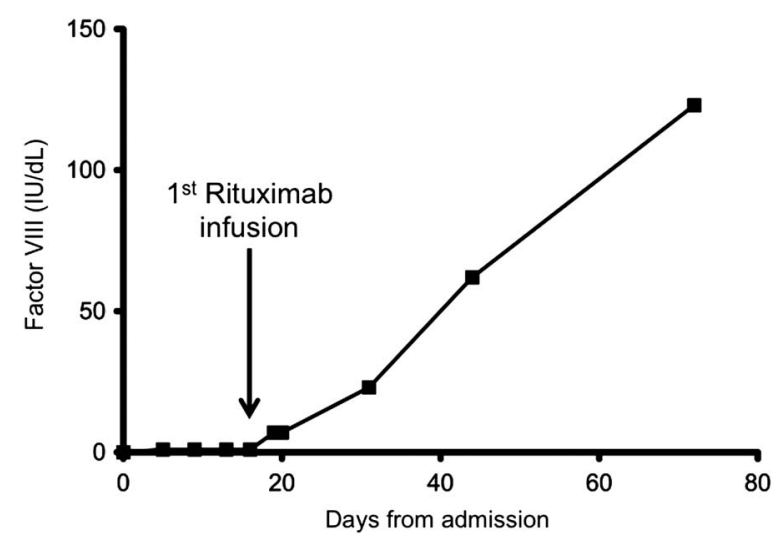

Figure 2 Increase in factor VIII levels in response to treatment with rituximab.

\section{DISCUSSION}

With an estimated $60 \%$ of the population believed to suffer an episode of epistaxis, the burden of this condition is significant. Despite this prevalence, only $10 \%$ of cases are believed to require medical input. ${ }^{1}$ Of the cases that are brought to medical attention, the majority are anterior bleeds and few require surgical intervention. ${ }^{2}{ }^{3}$ In the case presented, surgical intervention including endoscopic techniques could have been technically difficult and potentially detrimental in the context of a severe clotting disorder.

Acquired haemophilia is rare, with an incidence of 1.3-1.5 cases per million population per year in the UK. ${ }^{4} 5$ It is an autoimmune disease in which antibodies are generated against self factor VIII, resulting in depletion or reduced activity of this clotting factor. Although there is an association with pregnancy, the condition is predominantly seen in elderly patients with rheumatoid arthritis, malignancy or systemic lupus erythaematosus, or secondary to drug reactions. Despite these associations, many patients have no underlying systemic disease and no clear underlying cause. ${ }^{6}$ Similar to other disorders of coagulation, acquired haemophilia presents with unexplained ecchymosis, soft tissue haematomas, and postoperative or, more rarely, mucosal bleeding. ${ }^{7}$ Haemarthroses, common in hereditary haemophilia, are rare. As in this case, patients with acquired haemophilia often present with severe, sometimes lifethreatening haemorrhage, and the mortality rate is estimated to be between $7.9 \%$ and $22 \% .^{5} 68$

Laboratory diagnosis of acquired haemophilia requires a sequence of haematological tests as elevation of APTT with normal PT can result from von Willebrand disease (VWD), heparin use, lupus anticoagulant or deficiency of factors VIII, IX, XI or XII. In a patient not on heparin and whose APTT derangement is not erroneous, the first step in the diagnostic algorithm is screening for the inhibitor by mixing patient plasma with pooled normal plasma. Repeat APTT measurements are then performed both immediately and after incubation. Correction of APTT suggests VWD or factor deficiency and measurement of individual factors is performed to identify the specific deficit. Weak or no APTT correction indicates the presence of autoantibodies; hence a diagnosis of acquired haemophilia or lupus anticoagulant. Subsequent assays of intrinsic factors, inhibitor and lupus anticoagulant, are performed to specify the deficient factor and measure inhibitor load. In this case, although started on LMWH postoperatively following his hernia repair, at the time of presentation with epistaxis, the 
patient was not on heparin. Initial laboratory investigations demonstrated failure of APTT to correct with mixing. Coagulation factor assays demonstrated an isolated deficiency of factor VIII, diagnosing haemophilia, and a factor VIII inhibitor, confirming an autoimmune aetiology.

Alongside avoidance of bleeding precipitants, treatment of acquired haemophilia involves two processes-control of haemorrhage and inhibitor elimination. ${ }^{9}{ }^{10}$ In cases of severe bleeding and high inhibitor titre, as in this case, initial management aims at bypassing the deficient factor VIII. Bypassing agents are used to activate coagulation sufficiently to allow clotting and temporary cessation of haemorrhage. Options include rFVIIa, used here, and activated prothrombin complex concentrates (FEIBA: factor eight inhibitor bypassing activity). ${ }^{8}{ }^{11}$ However, it is not until elimination of the inhibitor is achieved that bleeding risk is reduced. ${ }^{10}$ Inhibitor elimination is orchestrated through immunosuppression, which should be initiated at diagnosis and is usually in the form of prednisolone either as a single agent or combined with cyclophosphamide. Where first line treatment fails, second line therapies include rituximab alone or in combination with other cytotoxics. 912

A review of the literature revealed four cases of acquired haemophilia associated with epistaxis; in three of these, the mucosal bleeding was a component of the coagulopathy presentation alongside subcutaneous and soft tissue haemorrhage. ${ }^{13-15}$ Only one case highlighted epistaxis as the primary complaint. ${ }^{16}$ Owing to the variety of bleeding sites associated with this

\section{Learning points}

- Vigilance with respect to clotting abnormalities and subsequent screening is crucial in the management of patients with recurrent epistaxis.

- Thorough history-taking and investigation with interspecialty liaison is vital in the identification and management of rare and challenging disorders.

- Beware attributing postoperative bleeding to the early use of prophylactic low molecular weight heparin in the setting of a deranged activated partial thromboplastin time. Repeat sampling can help diagnosis and prevent future life-threatening haemorrhage in cases of rare coagulopathies. condition at presentation, heightened awareness among multiple specialties is required to recognise this rare pathology and to implement multidisciplinary team management. Early haematology specialist input is crucial.

Contributors All the authors were involved in the care of the patient, the write-up and editing, and the review process of the manuscript.

Competing interests None declared.

Patient consent Obtained.

Provenance and peer review Not commissioned; externally peer reviewed.

\section{REFERENCES}

1 Petruson B, Rudin R. The frequency of epistaxis in a male population sample. Rhinology 1975;13:129-33.

2 Schlosser RJ. Clinical practice. Epistaxis. N Engl J Med 2009;360:784-9.

3 Kotecha B, Fowler $S$, Harkness $P$, et al. Management of epistaxis: a national survey. Ann R Coll Surg Engl 1996;78:444-6.

4 Collins $\mathrm{P}$, Macartney N, Davies $\mathrm{R}$, et al. A population based, unselected, consecutive cohort of patients with acquired haemophilia A. Br J Haematol 2004;124:86-90.

5 Collins PW, Hirsch S, Baglin TP, et al, UK Haemophilia Centre Doctors' Organisation. Acquired haemophilia A in the United Kingdom: a 2-year national surveillance study by the United Kingdom Haemophilia Centre Doctors' Organisation. Blood 2007;109:1870-7.

6 Green D, Lechner K. A survey of 215 non-hemophiliac patients with inhibitor to Facto VIII. Thromb Haemost 1981;45:200-3.

7 Hauser I, Schneider B, Lechner K. Post-partum factor VIII inhibitors. A review of the literature with special reference to the value of steroid and immunosuppressive treatment. Thromb Haemost 1995;73:1-5.

8 Hay CR, Negrier C, Ludlam CA. The treatment of bleeding in acquired haemophilia with recombinant factor VIla: a multicentre study. Thromb Haemost 1997:78:1463-7.

9 Hay CR, Brown S, Collins PW, et al. The diagnosis and management of factor VIII and IX inhibitors: a guideline from the United Kingdom Haemophilia Centre Doctors Organisation. Br J Haematol 2006;133:591-605.

10 Collins PW, Percy CL. Advances in the understanding of acquired haemophilia A: implications for clinical practice. Br J Haematol 2010;148:183-94.

11 Sallah S. Treatment of acquired haemophilia with factor eight inhibitor bypassing activity. Haemophilia 2004;10:169-73.

12 Collins P, Baudo F, Knoebl P, et al. Immunosuppression for acquired hemophilia A: results from the European Acquired Haemophilia Registry (EACH2). Blood 2012;120:47-55

13 Journet-Tollhupp J, Tchen T, Remy-Leroux V, et al. [Polymorphic eruption of pregnancy and acquired haemophilia A]. Ann Dermatol Venereol 2010;137:713-17.

14 Mansouritorghabeh $\mathrm{H}$, Lak M, van Heerde WL. Idiopathic factor VIII inhibitor autoantibody in a man presented after accident. Clin Appl Thromb Hemost 2009;15:588-90.

15 Horan P, Drake M, Patterson RN, et al. Acute onset dysphagia associated with an intramural oesophageal haematoma in acquired haemophilia. Eur J Gastroenterol Hepatol 2003;15:205-7.

16 Lalwani RB, Stricker RB. Case report: successful use of antifibrinolytic therapy in acquired factor VIII deficiency. Am J Med Sci 1992;303:398-401.

Copyright 2015 BMJ Publishing Group. All rights reserved. For permission to reuse any of this content visit

http://group.bmj.com/group/rights-licensing/permissions.

BMJ Case Report Fellows may re-use this article for personal use and teaching without any further permission.

Become a Fellow of BMJ Case Reports today and you can:

- Submit as many cases as you like

- Enjoy fast sympathetic peer review and rapid publication of accepted articles

- Access all the published articles

- Re-use any of the published material for personal use and teaching without further permission

For information on Institutional Fellowships contact consortiasales@bmjgroup.com

Visit casereports.bmj.com for more articles like this and to become a Fellow 